\title{
The Inflammatory Mediators and Sepsis
}

\author{
Adel M. Almawash* \\ Department of Botany and Microbiology, College of Sciences, \\ King Saud University, Riyadh, Saudi Arabia \\ *Corresponding author
}

\section{A B S T R A C T}

Keywords

Inflammatory mediators and

Sepsis,

Procalcitonin

Article Info

Accepted:

16 February 2018

Available Online:

10 March 2018
The occurrence, severity and mortality rates in human sepsis are increasing worldwide despite an increasing numerous clinical and experimental studies investigating sepsis. Sepsis is a severe clinical syndrome associated to the host response to infection. An auto-amplifying cytokine production: the cytokine storm is the main reason leading to the severity of infections. Cytokines are a large group of protein molecules that control the immune response during the inflammation in the body. Although, advancing in our knowledge about the processes that activate release great amount of cytokines, these understandings could not translate yet into effective treatments and diagnosis. This review aims to summarize our knowledge about most important inflammatory mediators in sepsis pathophysiology.

\section{Introduction}

Annually, many of patients are deaths due to infection diseases. Sepsis is leading causes of death every year with at least 19 million cases especially in low- and middle-income countries (Adhikari et al., 2010). The occurrence, severity and mortality rates in sepsis in humans are increasing globally, although the availability of immediate fluid resuscitation, antibiotics and improvement in intensive care unit, sepsis patients can rapidly deteriorate into septic shock-leading to multiple organ failure and death (Hotchkiss and Karl, 2003).
In the first days of the early acute inflammatory phase, some sepsis patients die. However, the majority of sepsis patients die after several days from secondary infections due to the immunosuppression. Many different immune cells, inflammatory mediators, and coagulation factors are involved in the pathophysiology of sepsis. Nevertheless, it is remains incompletely understood (Hotchkiss and Karl, 2003).

The virulence of the germ infections is not the main reason to cause damage in sepsis but also because of the host response after the 
detection of molecular patterns in the microorganisms [the pathogen-associated molecular patterns (PAMPs)]. Infection will cause a local reaction that will extend to the entire body mainly through the circulation of immune cells and soluble mediators (Chousterman, Swirski and Weber, 2017).

During the immune response, the different leukocytes and their metabolites are directed to kill the invading pathogen. Therefore, to provide the effective immune response without inflicting self-damage, the immune system needs to recognize and direct the appropriate immune response against the foreign invaders. The body to ensure appropriate responses has used many different mechanisms. However, this mechanism occasionally fails leading to severe tissue damage and death (Chousterman, Swirski and Weber, 2017).

In sepsis, cell and organ damage due to release of "inflammatory" cytokines that induce new cytokine production. This "cytokine storm" or "cytokine cascade" is responsible for the various signs associated with infection. Ultimately, a severe clinical syndrome, "sepsis" can be observed, when a threshold is crossed. It has a dramatic effect on morbidity and mortality (Chousterman, Swirski and Weber, 2017).

\section{Definition and epidemiology of sepsis}

In 1985, many of scientists hypothesized that sepsis is the host immune response to the invading pathogen. In 1992, the Society of Critical Care Medicine (SCCM) and the American College of Chest Physicians (ACCP) determine the criteria for sepsis and systemic inflammatory response syndrome (SIRS) due to the interchangeable use of terms, such as infection, bacteraemia, septicemia, sepsis etc., has led to confusion (Bone et al., 1992).
At that date sepsis was defined as a systemic inflammatory response syndrome (SIRS) due to infection and "Severe" sepsis was defined as sepsis associated with organ dysfunction, hypoperfusion, or hypotension. Also, "septic shock" was defined as sepsis with arterial hypotension in spite of adequate fluid resuscitation (Singer et al., 2016).

This definitions have been under discussion ever since and theses criteria were found to be unspecific. In 2001, the definitions were revisited sepsis as SIRS due to infection (assumed or confirmed) and severe sepsis as sepsis associated with acute organ dysfunction (Fig. 1). In 2016, a new definition of sepsis called Sepsis-3 and emerged to Sepsis is lifethreatening organ dysfunction due to a dysregulated host response to infection. This definition retuned the focus in the importance of organ dysfunction as a key diagnostic feature and highlighted the clinical findings in this syndrome. Therefore, unexplained acute organ failure in a critically ill patient should raise suspicion of possible sepsis. Moreover, and the quick Sequential Organ Failure Assessment (qSOFA) scoring system was advanced for the early identification of simultaneous organ dysfunction in sepsis (Gül et al., 2017).

Recent data regarding sepsis epidemiology show that sepsis continues to cause progressively more frequent and enduring problems in ICU's with high mortality, morbidity and hospital costs (Vincent, 2008). There is a great difference in sepsis occurrence throughout the world due to difference in the use of recommended definitions and a variance in local settings, and the full magnitude and impact of sepsis is not known (Gaieski et al., 2013). Annually, there are between 15 and 30 million of sepsis cases worldwide (Fleischmann et al., 2016) (Adhikari et al., 2010). Death from septic shock were often is about $80 \%$. However, 
with better surveillance and monitoring, support failing organs, advanced in training and therapy, mortality is now closer to 20-30 $\%$ in many series. Importantly, many of researchers have noted that sepsis patents that still not die until hospital discharge remain at risk for death in the following months and (Angus and van der Poll, 2013).

The majority of sepsis cases are caused by bacteria, whereas the viral, parasite and fungi being less frequent (Beale et al., 2009). However, study including 14,000 ICU septic patients in 75 countries reported that Gramnegative bacteria dominated then Grampositive bacterial and fungal infection(Vincent et al., 2009). It obviously appears that this cytokine storm-induced syndrome is a major public health issue. Unfortunately, In spite of advanced in sepsis understanding and studies, all clinical trials aiming to targeting the cytokines or dampen the inflammatory response failed (Chousterman, Swirski and Weber, 2017).

In this review, we will describe in detail the most important inflammatory mediator's role and their impact in sepsis pathophysiology

\section{Acute phase response and C-reactive protein in sepsis}

An acute phase response is triggered after pathogen recognition, leading to releasing a vast of proteins and some physiological changes including fever, altered lipid metabolism and activation of both coagulation and complement pathways. The major acute phase proteins includes C-reactive protein (CRP) and serum amyloid, induced by IL-1 or TNF $\alpha$ or IL-6 and play important role in antibacterial immunity (Sriskandan and Altmann, 2008).

Hepatocytes and other cells like alveolar macrophages, synthesize CRP. Initially, after the activation of liver cell by IL-6, the CRP is produced and elevated in $6 \mathrm{~h}$ after this activation (Sprung et al., 2008) and (Pepys and Hirschfield, 2003). Due to its ability to binds to several structures in bacterial surfaces, CRP has multiple functions including: facilitates bacterial opsonization and activates the complement system.

In healthy patients, CRP plasma concentration remains stable but its levels increase more than a thousand fold within a few days after immune activation such as trauma, inflammation, and other stimuli related to tissue damage. Therefore, CRP is a suitable biomarker for acute care situations (Sprung et al., 2008; Pepys and Hirschfield, 2003).

After Bacterial infections, CRP levels rapidly rise in a few hours due to IL-6 stimulation, also other cytokines (such as IL-1 and TNF-a) contribute to produceit. Alterations in plasma levels of CRP may be suitable in the diagnosis and prognosis of infection; a decrease in plasma levels indicates infection resolution and antimicrobial efficiency of treatment (Póvoa et al., 2005).

Nevertheless, CRP is quite unspecific because it is released regardless of infectious or noninfectious origin of inflammation. Therefore, Number of inflammatory conditions such as trauma, chronic inflammatory diseases and are associated with CRP production. Hence, CRP is not a specific indicator of bacterial infections. Consequently, sepsis progression is poorly correlated with changes in CRP level which makes CRP unreliable in sepsis diagnosis (Sprung et al., 2008) and (Pepys and Hirschfield, 2003).

On the other hand, CPR is characterized by its great sensitivity as soon as the inflammation is started and increased after $6 \mathrm{~h}$ of IL-6 activation with a half-life of 20-24 h (Hausfater, 2014; Mussap et al., 2013). 
Moreover, CRP laboratory tests are less expensive than cytokine measurements (Póvoa et al., 2005).

\section{Procalcitonin (PCT)}

PCT is a precursor peptide for the hormone calcitonin. Under homeostatic conditions, PCT is mainly produced in the thyroid, neuroendocrine cell lung and small intestine. In healthy individuals, the PCT Serum level are extremely low, $<0.05 \mathrm{ng} / \mathrm{ml}$, or even immeasurable. In particularly bacterial infections, systemic inflammation response distinguished by PCT secreted in huge quantities up to 1000 times. In addition, it is s released in a number of tissues (liver, lung, kidney, and adipose tissue) and immune cells. Moreover, it can be measured within 2-4 hours and reach the peak level within 6-24 hours of the stimulation (Mehanic and Baljic, 2013).

In addition, the level of CRP starts to reach a peak after 48 hours. On the other hand, PCT can be used as a marker of infection and sepsis because it is increased within 2-4 $\mathrm{h}$ from the start of the innate immunity cascade. Nonetheless, PCT reach a maximum value earlier than CRP. Thus, this kinetic profile is favorable and considered in the detection of infections at risk of rapid progression (Mussap et al., 2013) and (Reinhart et al., 2012) and (Becker, Snider and Nylen, 2008).

In addition, PCT concentration is not affected by immunodeficiency conditions, neutropenia and the use of steroid or non-steroid drugs, while the CRP is not stable. Furthermore, the concentration of PCT follows the severity of infection and the greatness of the inflammatory response. Therefore, the persistence of high values or the rise in PCT level is considered as prognostic indicator for severe forms of the disease with an adverse outcome (Mehanic and Baljic, 2013).
Actually, PCT is an effectiveness indicator of applied therapy, because its concentration is cut in halves 24 hours once controlling the infection, either with the immune system or a prescribed antibiotic, which reduce the use of antibiotics and cost of treatment (Mehanic and Baljic, 2013).

For both in inpatient and outpatient practice, when PCT is used in therapeutic guidelines, it helps to reduce the antibiotics usage without effects on the outcomes of the disease. In conclusion, once PCT is used in combination with other relevant laboratory parameters as a supplement to clinical observation of doctors, PCT has therapeutic and diagnostic significance in many indication areas (Mehanic and Baljic, 2013).

In comparing with all other presently available sepsis markers, PCT have potential for discriminating between infectious and noninfectious systemic inflammation (Harbarth et al., 2001). In addition, it may be capable to differentiate between viral and bacterial infections (Ahn et al., 2011).

In specific comparing between CRP and PCT, it has been found that PCT is particularly higher specificity and sensitivity for bacterial infection detection (Karlsson, 2015).

Several studies have reported that PCT could be used in distinguish between sepsis and SIRS of non-infectious origin, differentiate sepsis patients with or without bacterial origin, correlate the PCT level with severity of bacterial infection and aid to predict mortality in humans with sepsis. On the other hand, recent studies have been shown that PCT is increased in in a number of non-infectious conditions such as malignancies, trauma and pancreatitis, which limits its specificity to infection and sepsis (Karlsson, 2015). 
Nevertheless, PCT can be used to assess the progression of multi-organ failure and septic shock (Yaegashi et al., 2005). Two reports tried to found a PCT-algorithm that is beneficial to manage sepsis antibiotic treatment based on PCT serum concentration changes with inconsistent results. Jansen et al., showed that this algorithm correlated with the prolonged admission to the ICU without any development in the survival rate(Jensen $e t$ al., 2011), while Bouadma and colleagues showed this algorithm may decrease antibiotic exposure (Bouadma et al., 2010).

Many studies show that PCT value may distinguish Gram-positive from Gramnegative and fungal infections. Furthermore, Leli and colleagues show that that PCT cut-off of $\geq 10.8 \mathrm{ng} / \mathrm{mL}$ may suggest an infection by Gram-negatives and the $>1.3 \mathrm{ng} / \mathrm{mL}$ could be of help in ruling out a fungal bloodstream infection. A PCT cut-off $\leq 3.1 \mathrm{ng} / \mathrm{mL}$ could suggest exclusion of infection by Enterobacteriaceae. Nonetheless, using PCT to predict different microorganisms needs to be evaluated in further studies including detailed patient information (Leli et al., 2015).

\section{Cytokines in sepsis pathophysiology}

Cytokines are small proteins $(<40 \mathrm{kDa})$ that are broadly produced with the final aim of cell signaling (Dinarello, 2007). Cytokines can be divided into several categories: interleukins, interferon's, chemokine's, growth factors, and tumor necrosis factor. Furthermore, the most important group of cytokines is Interleukins that are secreted during infectious processes. They are released by leukocytes and endothelial cells and contribute to cell signaling and endorse activation, proliferation, death of immune cells (Chousterman et al., 2017).

Cytokines effects begin by binding to cell surface receptors. Subsequently, receptor activation causing activation of intracellular cascades, in the end leads to a bio- logical effect. A majority of cytokines is secreted in response to antigens but a minority of cytokines is constitutively expresses. Gene expression of cytokines is strongly regulated with rapid transcription and translation of proteins because of the facultative expression of these molecules (cytokines). For example, the production of pro-inflammatory cytokines such as IL-1 and IL-6 due to stimulation of transcription factor nuclear factor $\kappa \mathrm{B}(\mathrm{NF}-\kappa \mathrm{B})$ (Burkovskiy et al., 2013).

Indeed, cytokine play an important role in sepsis and are regulated by the network of pro-inflammatory cytokines (including TNF $\alpha$, IL$1 \beta$, IL-6, IL-8, IL-12 and IL-17), followed by release anti-inflammatory cytokines(including IL-1 receptor antagonist (IL1ra, IL-4, IL-10, IL-13, and TGF- $\beta$ ) (Devi Ramnath et al., 2006). Pro-inflammatory cytokines assistance to up regulates the immune system during cellular stressors or after antigens detection. While Anti-inflammatory cytokines modulate and decrease the immune response to return to homeostasis after antigens are eliminated (Burkovskiy et al., 2013).

For a long time, two phases of sepsis have been named: first phase named the systemic inflammatory response syndrome (SIRS), which is characterized by production of proinflammatory cytokines, from which the term "cytokine storm" arose (Aikawa, 1996). Afterward, SIRS phase is followed by a secondary phase called compensatory antiinflammatory response syndrome (CARS) with production of anti-inflammatory cytokines which restore immunological equilibrium (Tamayo et al., 2011). Immediately, the secretion of proinflammatory and immunosuppressive cytokines begins after onset of sepsis. To ensure the balance between these two phases, the host's need to maintain defense and 
minimizing self-induced tissue damage (Hotchkiss, Monneret and Payen, 2013). Consequently, the net effect of these two opposite responses is usually leading to hyperinflammatory phase and a secondary immunosuppressive phase (Manuscript, 2012).

\section{Pro-inflammatory cytokines}

Pro-inflammatory cytokines are fundamental in regulating the inflammation, trauma and the immune response to infection (

Table.1 Principal of pro-inflammatory cytokines including their sources, sites of action and their biological functions adapted of ). Because TNF- $\alpha$ and IL-1 play crucial role, they bind to target cells and activate production of more inflammatory mediators (Badiu et al., 2011) and (SiqueiraBatista et al., 2012) The production of a number of pro-inflammatory cytokines occurs simultaneously with generalized contribution of pathogen-responsive cells that results in sepsis (Gomes et al., 2015) (Fig. 2).

Many of experiments have been stated that cytokines are released in a sequential manner or a "cytokine cascade". After a stimulus induction, the cytokine cascade is initiated which lead to production of early or "proximal" cytokines such as TNF- $\alpha$ and IL1p. In addition to endotoxin, proximal cytokines activate the production of later or "distal" cytokines, including IL-6 and IL-8 (Blackwell and Christman, 1996).

Early cytokines including TNF- $\alpha$ and IL-1p mediate most of the physiological conflicts which are characteristic of sepsis(Blackwell and Christman, 1996). Specifically, IL-1 $\beta$ stimulates the amplification cascade and induces the synthesis of various inflammatory genes such as IL-6, IL-8 (Chousterman, Swirski and Weber, 2017).

During sepsis, the early cytokines are released within 30-90 minute period after exposure to stimulus. Interestingly, these cytokines are derived mainly from macrophage and share a remarkable array of biological effects. In addition, they are responsible for activating lipid mediators, late "distal" cytokines, reactive oxygen species (ROS), and cell adhesion molecules. Numerous studies have reported that IL- $1 \beta$ and TNF- $\alpha$ seems to be predictors of the severity of sepsis. However, using anti-TNF and anti-IL-1 $\beta$ to prevent death in septic patients are usefulness since the patient often comes to hospital at a late stage of disease and blocking these cytokines may be too late (Devi Ramnath et al., 2006).

Tumor Necrosis Factor Alpha (TNF $\alpha$ ) is a pro-inflammatory cytokine consist of a 17$\mathrm{kDa}$ protein released primarily by mononuclear phagocytes, which has pleiotrophic effects on target cells. TNF $\alpha$ is a main mediator of inflammation, and has been implicated in many of infectious and noninfectious inflammatory diseases (Blackwell and Christman, 1996). Moreover, this cytokine with IL-1 synergistically have primary function to promote inflammation by signaling pathways through specific receptors to stimulate gene expression of key inflammatory cytokines. Also, TNF $\alpha$ helps to induce the endothelial adhesion molecules to recruit other immune cells to fight the infection (Burkovskiy et al., 2013).

In addition, TNF- $\alpha$ is involved in the innate immune response and helps in macrophages and lymphocytes differentiation. The interaction between TNF- $\alpha$ and other cytokines endorses the production of proinflammatory effector molecules. Therefore, TNF- $\alpha$ has a significant function in coordinating the inflammatory response and activating the cytokines cascade (Gomes et al., 2015).

In several studies using recombinant TNF- $\alpha$ 
that is infused to human lead to SIRS with leucopenia, haemodynamic abnormalities, fever, coagulopathy and elevated liver enzymes. TNF- $\alpha$ is able to causing end-organ dysfunction which is shown in in severe sepsis. Many of experimental human and animal models of sepsis that induced by bacterial endotoxin show quickly activated of TNF- $\alpha$ in plasma within $60-90$ minutes after infusion (Figure 3). Furthermore, the concentration of TNF- $\alpha$ is correlated with severity of sepsis and TNF- $\alpha$ elevated is poor prognosis in sepsis patients. Moreover, the continue measurement of TNF- $\alpha$ may be better predictors of outcome than single measurement (Blackwell and Christman, 1996).

Table.1 Principal of pro-inflammatory cytokines including their sources, sites of action and their biological functions adapted of (Gomes et al., 2015)

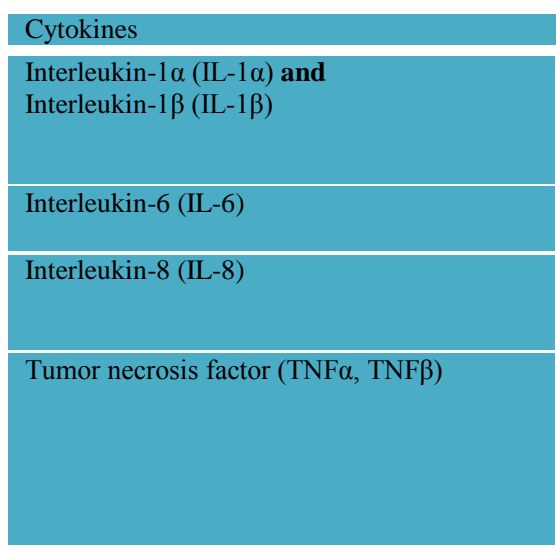

\begin{tabular}{|l|l|}
\hline $\begin{array}{l}\text { Secreted By } \\
\text { Macrophages, dendritic cells, } \\
\text { fibroblasts, endothelial cells, } \\
\text { keratinocytes, hepatocytes. }\end{array}$ & $\begin{array}{l}\text { Eites of Action and Biological Effects } \\
\text { coagulation) } \\
\text { Hypothalamus: fever } \\
\text { Liver: synthesis of acute phase proteins }\end{array}$ \\
\hline Macrophages, endothelial cells, T cells & $\begin{array}{l}\text { Liver: synthesis of acute phase proteins } \\
\text { B cells: proliferation of antibody-producing cells }\end{array}$ \\
\hline $\begin{array}{l}\text { monocytes, macrophages, neutrophils, } \\
\text { and intestine, kidney, placenta, and } \\
\text { bone marrow cells }\end{array}$ & $\begin{array}{l}\text { It is a chemotactic factor that attracts neutrophils, } \\
\text { basophils, and T-cells during the inflammatory } \\
\text { process m and involved in neutrophil activation }\end{array}$ \\
\hline Macrophages, NK cells, T cells & $\begin{array}{l}\text { Endothelial cells: activation (inflammation, } \\
\text { coagulation) } \\
\text { Neutrophils: activation } \\
\text { Hypothalamus: fever } \\
\text { Liver: synthesis of acute phase proteins } \\
\text { Muscles, fat: catabolism (cachexia) }\end{array}$ \\
\hline
\end{tabular}

Fig.1 SIRS and CARS. After onset of sepsis, the pro-inflammatory immune response and the anti-inflammatory immune response begin activation. During the first days, the majority of patients survive in this phase. However, the death is occurred due to the hyper-inflammatory immune response. If the sepsis continues, patients may die in immunosuppression phase due to secondary infections. Adapted from Hotchkiss, Monneret and Payen (2013) 


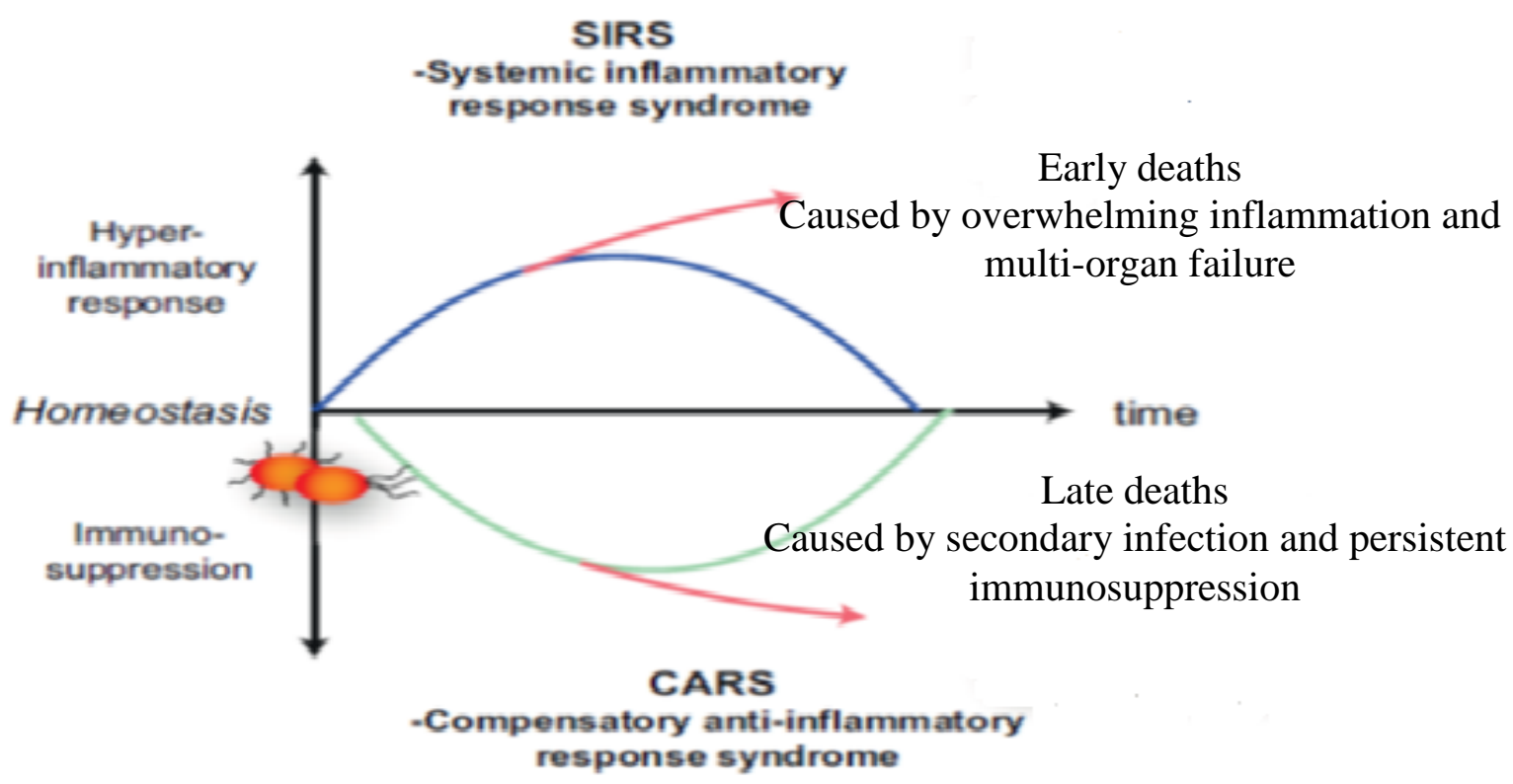

Fig.2 Sepsis response including two phases: an early pro-inflammatory phase followed by a later anti-inflammatory phase. When both of these phases are balanced, an ideal response occurs. (A)

An early excessive response lead to early mortality associated shock. On the other hand, An exaggerated later response lead to immunosuppression or immunoparalysis with multi- infections and multi- organ failure (B) (Webster and Galley, 2009) 

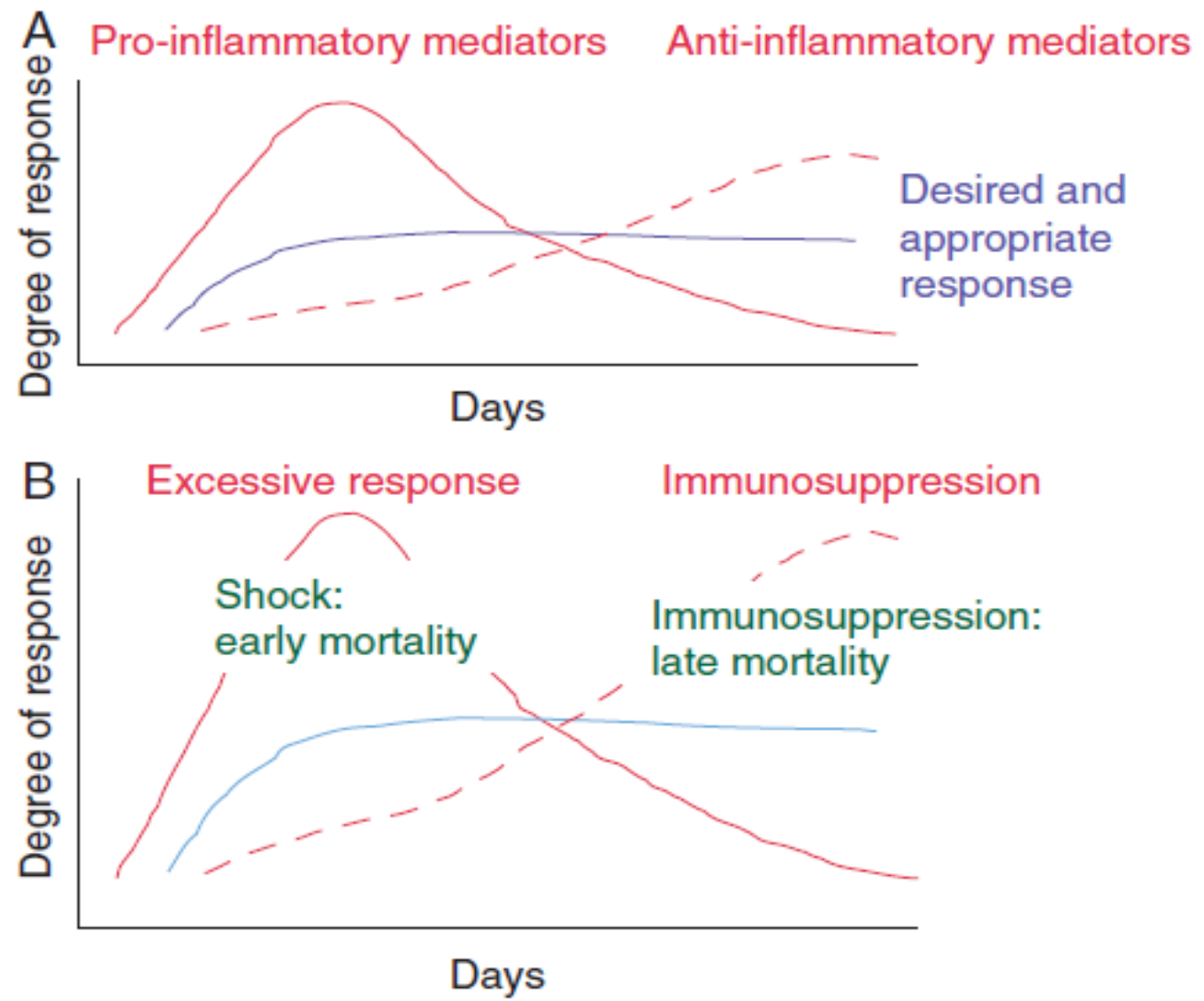

Fig.3 Pro-inflammatory and anti-inflammatory cytokine production during sepsis. Adapted from Boontham et al., (2003)

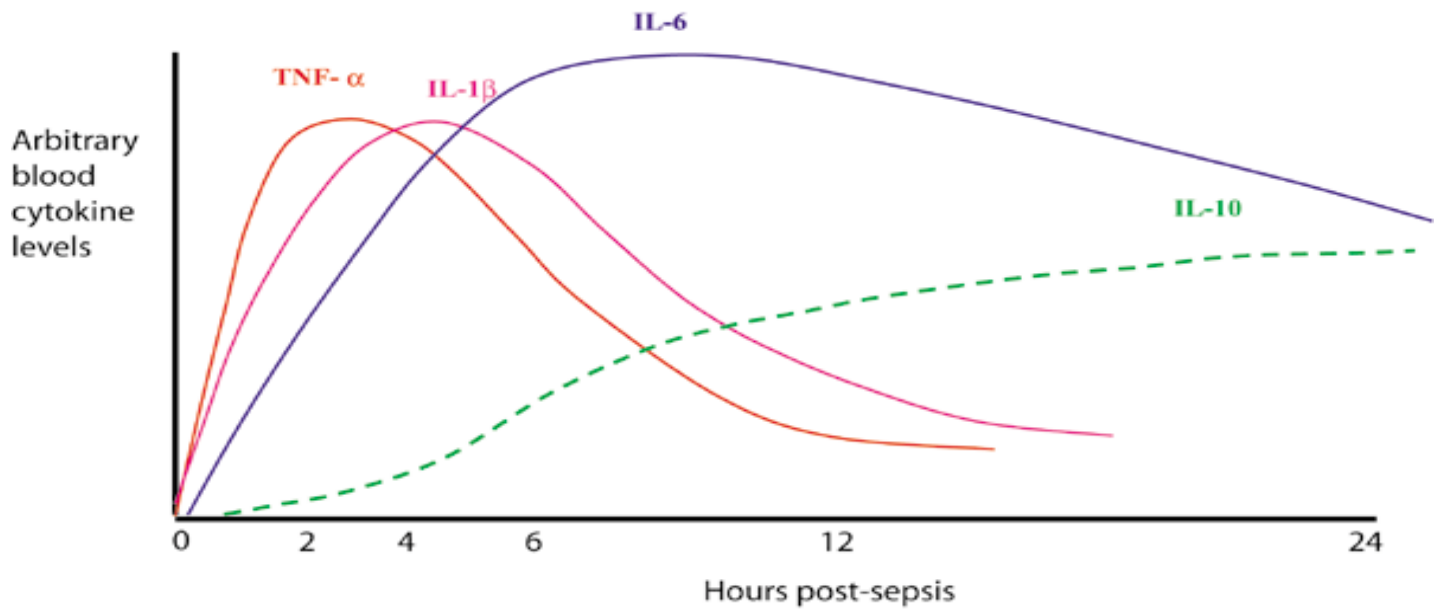

Interleukin 1 (IL-1) is synthesized by polymorphonuclear, mononuclear and other cell types and affects several of tissues. IL-1 is named to proteins IL-1 $\alpha$ and IL1 $\beta$ which activate the same IL-1 receptors and therefore share the same biological activates. Also, they share many of biological activities of TNF- $\alpha$. Nevertheless, IL-1 $\beta$ is the predominant form of this cytokine released from stimulated monocyte and detected in plasma septic patients. After infusion of endotoxin, IL-1 $\beta$ is increased in human. Similarly to TNF- $\alpha$, IL-1 
$\beta$ stimulates the release the other cytokines including IL-6, IL-8 and TNF- $\alpha$ (Blackwell and Christman, 1996).

Further studies have been detected IL- $1 \beta$ in plasma of sepsis patients and it is not normally present in human plasma. IL-1 $\beta$ reaches the peak after 4 hour of endotoxin injection and remain elevated in non-survival patients for 22 hour and subsequently return to normal in survivors. In conclusion, IL-1 $\beta$ is increased in plasma of human sepsis patients and there is a correlation between the concentration of IL-1 $\beta$ and the severity of sepsis but there is no convincing association with mortality (Blackwell and Christman, 1996).

Late pro-inflammatory cytokines including IL-6 and IL-8 reaches a peak concentration after TNF- $\alpha$ and IL-1 $\beta$ reaches a peak (Fig.3 Pro-inflammatory and anti-inflammatory cytokine production during sepsis. Adapted from Boontham et al., (2003)). In more detail, IL-6 is $21-\mathrm{kDa}$ glycoprotein released mainly by the tissue macrophages and by several cell types including lymphocytes, fibroblast, and endothelial cells in response to endotoxin and early cytokines such as TNF- $\alpha$ (Devi Ramnath et al., 2006). Actually, IL-6 has several biological effects including induction of acute phase protein, stimulation of B- and T-lymphocytes, modulation of hematopoiesis, and functions as pyrogens (Blackwell and Christman, 1996). Furthermore, IL-6 is classified as both a proinflammatory and anti-inflammatory cytokine and is linked with bacterial sepsis (Burkovskiy et al., 2013).

Kuhus and colleagues found that after activation by endotoxin injection into healthy human volunteers, plasma IL-6 peaked at 4 hours (Blackwell and Christman, 1996). Interestingly, high levels of IL-6 were noted in many inflammatory diseases such as autoimmune diseases, cardiovascular disease, or cancer. In sepsis, the appearance of plasma IL- 6 may be related directly with TNF- $\alpha$ and IL- $1 \beta$ production and the exact role of IL- 6 in sepsis are unclear and maybe related to capillary leakage and the activation of the complement pathway (Riedemann et al., 2004) and (Riedemann et al., 2003). Similarly to IL-1 $\beta$, high levels of IL- 6 were consistently observed to be related with the severity of sepsis and the highest levels related with the worse outcome (Gouel-Chéron et al., 2012), (Wu et al., 2009) and (Kellum et al., 2007). According to the several studies of IL-6 in human disease, simply the concentration of IL-6 in sepsis seems to be a good marker of the initiation of the cytokine cascade and may reflect the association of host inflammatory response and sepsis severity. (Blackwell and Christman, 1996).

Finally, plasma IL-6 measurements have still not been integrated into the routine clinical investigations, although there have been efforts to develop an IL-6 assay approved by the FDA and the plasma IL-6 measurements appeared to be promising. The main reason in the failure to use IL- 6 measurement routinely in the clinical setting has more to do with the interpretation of the results, and their value when compared to existing biomarkers.

Currently, IL-6 is used to evaluate the severity of the inflammatory response. Furthermore, the general consensus by most clinicians is that existing diagnostics, such as CRP, total and differential white blood cell count, and the clinical condition of the patient are sufficient to judge the severity of the inflammatory insult and there is no compelling need to add an additional biomarker (Gentile et al., 2013).

IL-8 is a small, basic protein produced by many of cells including endothelial, phagocytes and mononuclear cells response to 
multiple of stimuli including endotoxin and early cytokines.

The primary functions of IL-8 are chemoattract and stimulate neutrophils to the site of inflammation functioning as a chemical signal that draws neutrophils to the site of inflammation (Gomes et al., 2015). Moreover, IL-8 has been reported in bronchoalveolar lavage fluids of patients with acute respiratory distress syndrome (ARDS) and contributed in the multiple organ dysfunction syndromes (Miller, Cohen and Matthay, 1996).

In human sepsis, IL-8 production same as IL6 is activated by TNF- $\alpha$. Nonetheless, many of studies show that the level of IL-8 Is elevated in patents with sepsis and this elevation is correlated with mortality rate. Several studies indicate that the main role of IL-8 in sepsis is activating and recruiting neutrophils to the site of infection which can result in tissue injury (Blackwell and Christman, 1996).

During sepsis, IL-12 is also elevated and released by macrophages, dendritic cells, and lymphoblastoid cells. The main function of this cytokine is activating $\mathrm{NK}$ cells and inducing the differentiation of naïve $\mathrm{T}$ cells into type 1 helper $\mathrm{T}$ cells (TH1); consequently, a high amount of INF $\gamma$ is released of these cells (Hsieh et al., 1993).

\section{Anti- inflammatory cytokines}

The most studied anti-inflammatory interleukins are IL- 1RA, IL-4, and IL-10. Nonetheless, anti-inflammatory cytokines are associated with the slowing of responses to causative agents, creating a compensatory anti-inflammatory response syndrome (CARS). Anti-inflammatory cytokines are more released when the patient survives suffering of the disorders associated to systemic inflammation (Gomes et al., 2015).

IL-1RA is released by immune cells or epithelial cells and able to blocking the action of IL- $1 \alpha$ or IL- $1 \beta$ inflammatory signals due to its ability to bind to IL-1R (Dinarello, 1998).

IL-4 is predominantly released by $\mathrm{TH} 2$ and capable to stimulate both proliferation of $\mathrm{T}$ and $\mathrm{B}$ cells and shift of T cells towards a TH2 profile (Wynn, 2015) and (Luzina et al., 2012). Furthermore, IL-4 contributes to a very powerful downregulationof soluble and cellular pro-inflammatory activities. The role of IL-4 In sepsis is unclear. However, numerous of reports illustrated its pathophysiology in sepsis (Couper, Blount and Riley, 2008).

IL-10 is the central anti-inflammatory cytokine and produced by several of immune cells including: dendritic cells, macrophages, B-lymphocytes, T-regulatory cells (TREGS), and natural killer $\mathrm{T}$ cells (NKT cells). Therefore, IL-10 helps modulate the proinflammatory cytokines production during early stages of sepsis by making targets more tolerant to repeated pro-inflammatory stimuli and limiting pro- inflammatory effects. Recent reports have confirmed that IL-10 is demonstrable in plasma of sepsis patient and there is significant elevation in IL-10 in septic patient than in patient with sepsis alone (Blackwell and Christman, 1996). Many of other studies indicate that the main role of IL10 in sepsis may be based on the patient immune status. Thus, involving this cytokine is time critical during sepsis pathogenesis to give effective treatments (Burkovskiyet al., 2013).

In addition, a large amount of data has advised that IL-10 is a candidate for treating bacteria sepsis. Consequently, it has been reported that patients who die of sepsis do not release IL-10 and it reduces the mortality in various endotoxic or septic shock models (Devi Ramnath et al., 2006).

The host response to infection agent is still 
incompletely understood. Moreover, it has become widely accepted that sepsis is a complex and non-linear process. Furthermore, sepsis occurs when the inflammatory mediators are excessively released to eradicate the invader and restore homeostasis that called auto-amplifying phenomenon. However, we still lack a comprehensive integrative view, at the cell level, in time and space. Therefore, the next decade will probably see a more precise depiction of incell, in-organ, and global response to infection.

While the exact triggers that should be targeted are still to be identified, many of failed trials aim to damp this excessive cytokines activation or its consequences.In addition, there is need to develop new diagnostic tools to be more precise determination of the immune/ inflammatory status during sepsis, and may contribute in patients therapeutic strategy.

In clinical practice, there has yet to be a way to prospectively use the cytokines profiles, although researchers have been capable to distinct cytokine profiles associated with sepsis severity, organ failure, and mortality. Multitude of studies both in support of and against the usefulness of cytokines as prognostic biomarkers mostly hampered by small sample sizes, variable patient populations, and heterogeneous biomarker assays.

Multiplex cytokine approaches have been employed without to be accepted to be in clinical practice, only Procalcitonin has probability of entering the routine clinical practice in the immediate future. Nevertheless, Procalcitonin has been used for the identification of sepsis and to guide the discontinuation of antibiotic therapy when levels are not raised.

\section{References}

Adhikari, N. K. J. et al., (2010) 'Critical care and the global burden of critical illness in adults', The Lancet, 376(9749), pp. 13391346. doi: 10.1016/S0140-6736(10)604461.

Ahn, S. et al., (2011) 'Role of procalcitonin and C-reactive protein in differentiation of mixed bacterial infection from 2009 H1N1 viral pneumonia', Influenza and other Respiratory Viruses, 5(6), pp. 398-403. doi: 10.1111/j.1750-2659.2011.00244.x.

Aikawa, N. (1996) '[Cytokine storm in the pathogenesis of multiple organ dysfunction syndrome associated with surgical insults].', Nihon Geka Gakkai zasshi, 97(9), pp. 771-7. Available at: http://www.ncbi. nlm.nih.gov/pubmed/8940690.

Angus, D. C. and van der Poll, T. (2013) 'Severe sepsis and septic shock.', The New England journal of medicine, 369(9), pp. 840-51. doi: 10.1056/NEJMra1208623.

Badiu, D. C. et al., (2011) 'Proinflammatory cytokines in peritonitis.', Journal of medicine and life, 4(2), pp. 158-62. Available at: http://www.pubmedcentral.nih.gov/articlere nder. .fcgi $?$ artid $=3124274 \&$ tool $=$ pmcentrez \&rendertype $=$ abstract.

Beale, R. et al., (2009) 'Promoting global research excellence in severe sepsis (PROGRESS): Lessons from an international sepsis registry', Infection, 37(3), pp. 222-232. doi: 10.1007/s15010-008-8203-Z.

Becker, K. L., Snider, R. and Nylen, E. S. (2008) 'Procalcitonin assay in systemic inflammation, infection, and sepsis: Clinical utility and limitations', Critical Care Medicine, 36(3), pp. 941-952. doi: 10.1097/CCM.0B013E318165BABB.

Blackwell, T. S. and Christman, J. W. (1996) 'Sepsis and cytokines: current status', British Journal of Anaesthesia, 77(1), pp. 110-117. doi: 10.1093/bja/77.1.110.

Bone, R.C., Balk, R.A., Cerra, F.B., et al., (1992) 'Definitions for sepsis and organ failure and accplsccm consensus conference', Che, 101(6), pp. 1644-1655. doi: 10.1378/chest.101.6.1644.

Boontham, P. et al., (2003) 'Surgical sepsis: 
Dysregulation of immune function and therapeutic implications', Surgeon, pp. 187-206. doi: 10.1016/S1479666X(03)80018-5.

Bouadma, L. et al., (2010) 'Use of procalcitonin to reduce patients' exposure to antibiotics in intensive care units (PRORATA trial): a multicentre randomised controlled trial', The Lancet. Elsevier Ltd, 375(9713), pp. 463-474. doi: 10.1016/S01406736(09)61879-1.

Burkovskiy, I. et al., (2013) 'Cytokine release in sepsis', Adv Bioscience Biotech, 2013(4), pp. 860-865. doi: 10.4236/abb.2013.49114.

Chousterman, B. G., Swirski, F. K. and Weber, G. F. (2017) 'Cytokine storm and sepsis disease pathogenesis', Seminars in Immunopathology, pp. 517-528. doi: 10.1007/s00281-017-0639-8.

Couper, K. N., Blount, D. G. and Riley, E. M. (2008) 'IL-10: The Master Regulator of Immunity to Infection', The Journal of Immunology, 180(9), pp. 5771-5777. doi: 10.4049/jimmunol.180.9.5771.

Devi Ramnath, R. et al., (2006) 'Inflammatory mediators in sepsis: Cytokines, chemokines, adhesion molecules and gases', Journal of Organ Dysfunction, pp. 80-92. doi: 10.1080/17471060500435662.

Dinarello, C. A. (1998) 'Interleukin-1, Interleukin-1 Receptors and Interleukin-1 Receptor Antagonist', International Reviews of Immunology, 16(5-6), pp. 457499. doi: 10.3109/08830189809043005.

Dinarello, C. A. (2007) 'Historical insights into cytokines', European Journal of Immunology. doi: 10.1002/eji.200737772.

Fleischmann, C. et al., (2016) 'Assessment of global incidence and mortality of hospitaltreated sepsis current estimates and limitations', American Journal of Respiratory and Critical Care Medicine, 193(3), pp. 259-272. doi: 10.1164/rccm.201504-07810C.

Gaieski, D. F. et al., (2013) 'Benchmarking the Incidence and Mortality of Severe Sepsis in the United States*', Critical Care Medicine, 41(5), pp. 1167-1174. doi: 10.1097/CCM.0b013e31827c09f8.

Gentile, L. F. et al., (2013) 'Is there value in plasma cytokine measurements in patients with severe trauma and sepsis?', Methods (San Diego, Calif.), 61(1), pp. 3-9. doi: 10.1016/j.ymeth.2013.04.024.

Gomes, A. P. et al., (2015) 'Pro-Inflammatory Cytokines in Sepsis: Biological Studies and Prospects From In Silico Research', Biological Systems: Open Access, 5(1), pp. 1-7. doi: 10.4172/2329-6577.1000158.

Gouel-Chéron, A. et al., (2012) 'Early interleukin6 and slope of monocyte human leukocyte antigen-DR: A powerful association to predict the development of sepsis after major trauma', PLoS ONE, 7(3). doi: 10.1371/journal.pone.0033095.

Gül, F. et al., (2017) 'Changing definitions of sepsis', Turk Anesteziyoloji ve Reanimasyon Dernegi Dergisi, pp. 129138. doi: 10.5152/TJAR.2017.93753.

Harbarth, S. et al., (2001) 'Diagnostic value of procalcitonin, interleukin-6, and interleukin-8 in critically ill patients admitted with suspected sepsis', American Journal of Respiratory and Critical Care Medicine, 164(3), pp. 396-402. doi: 10.1164/ajrccm.164.3.2009052.

Hausfater, P. (2014) 'Biomarkers and infection in the emergency unit', Medecine et Maladies Infectieuses. Elsevier Masson SAS, 44(4), pp. 139-145. doi: 10.1016/j.medmal.2014.01.002.

Hotchkiss, R. S. and Karl, I. E. (2003) 'The pathophysiology and treatment of sepsis.', The New England journal of medicine, 348(2), pp. 138-50. doi: 10.1056/NEJMra021333.

Hotchkiss, R. S., Monneret, G. and Payen, D. (2013) 'Sepsis-induced immuno suppression: From cellular dysfunctions to immunotherapy', Nature Reviews Immunology. Nature Publishing Group, 13(12), pp. 862-874. doi: 10.1038/nri3552.

Hsieh, C. et al., (1993) 'Development of TH1 CD4+ $\mathrm{T}$ cells through IL-12 produced by Listeria-induced macrophages', Science, 260(5107), pp. 547-549. doi: 10.1126/science.8097338.

Jensen, J. U. et al., (2011) 'Procalcitonin-guided interventions against infections to increase early appropriate antibiotics and improve survival in the intensive care unit: A randomized trial', Critical Care Medicine, 
39(9), pp. 2048-2058. doi: 10.1097/CCM. $0 \mathrm{~b} 013 \mathrm{e} 31821 \mathrm{e} 8791$.

Karlsson, I. (2015) Cytokines as Diagnostic Biomarkers in Canine Pyometra and Sepsis.

Kellum, J. A. et al., (2007) 'Understanding the inflammatory cytokine response in pneumonia and sepsis: Results of the genetic and inflammatory markers of sepsis (GenIMS) study', Archives of Internal Medicine, 167(15), pp. 1655-1663. doi: 10.1001/archinte.167.15.1655.

Leli, C. et al., (2015) 'Procalcitonin levels in gram-positive, gram-negative, and fungal bloodstream infections', Disease Markers, 2015. doi: $10.1155 / 2015 / 701480$.

Luzina, I. G. et al., (2012) 'Regulation of inflammation by interleukin-4: a review of "alternatives", Journal of Leukocyte Biology, 92(4), pp. 753-764. doi: 10.1189/jlb.0412214.

Manuscript, A. (2012) 'Advance in the Management of Sepsis and $\mathrm{i} n$ the Understanding of Key Immunologic Defects of the Disorder', Anesthesiology, 115(6), pp. 1349-1362. doi: 10.1097/ALN. 0b013e31823422e8.Advances.

Mehanic, S. and Baljic, R. (2013) 'The Importance of Serum Procalcitonin in Diagnosis and Treatment of Serious Bacterial Infections and Sepsis', Materia Socio Medica, 25(4), p. 277 . doi: 10.5455/msm.2013.25.277-281.

Miller, E. J., Cohen, A. B. and Matthay, M. A. (1996) 'Increased interleukin-8 concentrations in the pulmonary edema fluid of patients with acute respiratory distress syndrome from sepsis.', Critical care medicine, 24(9), pp. 1448-54. Available at: http://www.ncbi.nlm.nih. gov/pubmed/8797614.

Mussap, M. et al., (2013) 'The importance of biomarkers in neonatology', Seminars in Fetal and Neonatal Medicine. Elsevier Ltd, 18(1), pp. 56-64. doi: 10.1016/j.siny.2012.10.006.

Pepys, M. B. and Hirschfield, G. M. (2003) 'Creactive protein: a critical update.', The Journal of clinical investigation, 111(12), pp. 1805-1812. doi: 10.1172/JCI18921.

Póvoa, P. et al., (2005) 'C-reactive protein as a marker of infection in critically ill patients.', Clinical microbiology and infection: the official publication of the European Society of Clinical Microbiology and Infectious Diseases, 11(2), pp. 101108. doi: 10.1111/j.1469-0691.2004. 01044.x.

Reinhart, K. et al., (2012) 'New approaches to sepsis: Molecular diagnostics and biomarkers', Clinical Microbiology Reviews, 25(4), pp. 609-634. doi: 10.1128/CMR.00016-12.

Riedemann, N. C. et al., (2003) 'Protective Effects of IL-6 Blockade in Sepsis Are Linked to Reduced C5a Receptor Expression', The Journal of Immunology, 170(1), pp. 503-507. doi: 10.4049/jimmunol.170.1.503.

Riedemann, N. C. et al., (2004) 'Regulatory role of C5a in LPS-induced IL-6 production by neutrophils during sepsis.', The FASEB journal: official publication of the Federation of American Societies for Experimental Biology, 18(2), pp. 370-372. doi: 10.1096/fj.03-0708fje.

Singer, M. et al., (2016) 'The third international consensus definitions for sepsis and septic shock (sepsis-3)', JAMA - Journal of the American Medical Association, pp. 801810. doi: 10.1001/jama.2016.0287.

Siqueira-Batista, R. et al., (2012) 'CD4+CD25+ T lymphocytes and regulation of the immune system: Perspectives for a pathophysiological understanding of sepsis', Revista Brasileira de Terapia Intensiva, 24(3), pp. 294-301. doi: 10.1590/S0103-507X2012000300014.

Sprung, C. L. et al., (2008) 'Hydrocortisone therapy for patients with septic shock.', The New England journal of medicine, 358(2), pp. 111-24. doi: 10.1056/NEJMoa071366.

Sriskandan, S. and Altmann, D. M. (2008) 'The immunology of sepsis', Journal of Pathology, pp. 211-223. doi: 10.1002/ path.2274.

Tamayo, E. et al., (2011) 'Pro- and antiinflammatory responses are regulated simultaneously from the first moments of septic shock', European Cytokine Network, 22(2), pp. 82-87. doi: 10.1684/ecn.2011.0281. 
Vincent, J. et al., (2009) 'International study of the prevalence and outcomes of infection in intensive care units.', JAMA, 302(21), pp. 2323-9. doi: 10.1001/jama.2009.1754.

Vincent, J.-L. (2008) 'Clinical sepsis and septic shock--definition, diagnosis and management principles.', Langenbeck's archives of surgery / Deutsche Gesellschaft fur Chirurgie, 393(6), pp. 817-824. doi: 10.1007/s00423-008-0343-1.

Webster, N. R. and Galley, H. F. (2009) 'Immunomodulation in the critically ill', British Journal of Anaesthesia, 103(1), pp. 70-81. doi: 10.1093/bja/aep128.

Wu, H.-P. et al., (2009) 'Serial cytokine levels in patients with severe sepsis.', Inflammation research: official journal of the European Histamine Research Society... [et al.,], 58(7), pp. 385-93. doi: 10.1007/s00011009-0003-0.

Wynn, T. A. (2015) 'Type 2 cytokines: Mechanisms and therapeutic strategies', Nature Reviews Immunology, pp. 271-282. doi: $10.1038 /$ nri3831.

Yaegashi, Y. et al., (2005) 'Evaluation of a newly identified soluble CD14 subtype as a marker for sepsis', Journal of Infection and Chemotherapy, 11(5), pp. 234-238. doi: 10.1007/s10156-005-0400-4.

\section{How to cite this article:}

Adel M. Almawash. 2018. The Inflammatory Mediators and Sepsis. Int.J.Curr.Microbiol.App.Sci. 7(03): 1763-1776. doi: https://doi.org/10.20546/ijcmas.2018.703.208 